\title{
Risk of retinal detachment following cataract extraction: results from the International Cataract Surgery Outcomes Study
}

\author{
Jens Christian Norregaard, Henrik Thoning, Tavs Folmer Andersen, Peter \\ Bernth-Petersen, Jonathan C Javitt, Gerald F Anderson
}

Department of Social Medicine, University of Copenhagen, Denmark

J C Norregaard

T F Andersen

Danish Institute of Clinical Epidemiology, Copenhagen, Denmark $\mathrm{H}$ Thoning

Ophthalmological Department, Hvidovre University Hospital, Copenhagen, Denmark J C Norregaard

$P$ Bernth-Petersen

Worthen Center for Eye Care Research, Georgetown

University,

Wahington, DC, USA

J C Javitt

Department of Health

Policy and

Management, Johns

Hopkins University,

Baltimore, USA

G F Andersen

Correspondence:

Jens Christian Norregaard

MD, Department of Socia

MD, Department of Sociane, University of

Medicine, University of

DK 2200 Copenhagen $\mathrm{N}$ Denmark.

Accepted for publication 1 April 1996

\begin{abstract}
Aims-To estimate the risk of retinal detachment (RD) following cataract extraction in Denmark, and to compare the risk with that following cataract extraction in the USA, and with that in a sample of Danish patients who did not have ocular surgery.

Methods-A sample was created from the administrative Danish Hospital Register and included 19252 patients who underwent first eye cataract surgery between 1985 and 1987, and who were 50 years of age or older. The patients were then followed for 4-6 years using the register data. The design and definition of events were identical to the US National Study of Cataract Outcomes.

Results-In Denmark a 4 year cumulative risk of hospitalisation for RD of $0.93 \%$ (95\% confidence interval (CI) 0.71-1.16) was observed following an extracapsular cataract extraction with a lens implant. A similar cumulative risk of RD was reported from the US study. Thus, no difference in outcomes concerning risk of RD was shown between Denmark and the USA. In a multivariate analysis younger age, male sex, and intracapsular cataract extraction were all associated with higher risk of postoperative RD. A reference group of 7636 people not undergoing any ocular surgery was created and the incidence of RD in this group was calculated. During the sixth year following cataract surgery, the incidence of $R D$ in the cataract group was still $7.5 \quad(95 \%$ CI 1.6-22.0) times higher than that observed in the reference group.

(Brf Ophthalmol 1996;80:689-693)
\end{abstract}

Retinal detachment (RD) following cataract extraction is a rare complication but when it occurs it is likely to threaten the visual outcome. Consequently, knowledge about the risk factors for $\mathrm{RD}$ and reliable estimates of the risk of $\mathrm{RD}$ are important to the cataract surgeon. However, most studies of RD after cataract surgery are based on patients treated by individual surgeons or at academic centres. ${ }^{1-6}$ Such series do not necessarily reflect complication rates in the overall health care system and may not be generalised.

We calculated the risk of $\mathrm{RD}$ after cataract surgery in the Danish public health care system based on data from an administrative database. Such nationwide administrative databases can provide large sample sizes but they also have the advantage of showing the outcomes of treatment for a cross section of the population. As a cross national comparison can be a powerful method when assessing wider applicability of national clinical results, the Danish study was designed and carried out in close collaboration with the US National Study of Cataract Outcomes. ${ }^{7}$ We kept the definition of events and the method of data analysis in the present Danish study as close as possible to those used in the US study to maximise the comparability of the results. It is therefore possible for us subsequently to compare the Danish results to those previously reported from the US National Study. ${ }^{7}$ The results from the Danish cataract group were also compared with a reference group of people who did not undergo intraocular surgery. This was done to investigate whether an elevated risk of $\mathrm{RD}$ in the cataract group existed throughout the observation period or only during the first few years after the cataract surgery.

The present study is part of the International Cataract Surgery Outcomes Study sponsored by the Agency for Health Care Policy and Research, US Department of Health and Human Services. This project consists of a series of studies, which examine and compare outcomes of cataract surgery in different health care environments.

\section{Materials and methods}

DANISH SAMPLE OF CATARACT PATIENTS

We performed a retrospective cohort study based on the administrative hospital register of the National Board of Health called the Danish National Patient Register (DNPR). After each admission to a public health service hospital, a discharge summary is created which summarises diagnoses and surgical procedures performed during that admission. The corresponding International Classification of Diseases (ICD) codes for diagnoses and surgical procedures are routinely reported to DNPR ${ }^{8}$ Every Danish citizen has been assigned a personal identification number that is used by all public authorities including the DNPR. Using this number as a key to linkage, it is possible to identify, obtain, and aggregate longitudinal, person specific information from various public registers.

We identified all patients age 50 years or older who underwent cataract extraction on an 
inpatient basis between 1985 and 1987 with 4-6 years of follow up. Any admission coded for cataract extraction was identified. The admission during which a cataract extraction was performed is denoted as the index admission. About $5 \%$ of the cataract surgery performed in Denmark between 1985 and 1987 was done on an outpatient basis or in private clinics ${ }^{9}$ and is not included in our present sample. If two cataract extractions were identified for a single patient during the study period, the first occurrence was chosen as the index admission. We excluded all cases of cataract surgery that were combined with other ocular procedures such as corneal graft, glaucoma, or posterior segment procedures. Patients were also excluded if they had a code for 'previous RD procedures' applied to the index admission. Of a total sample of 19539 patients, we excluded 287 patients based on these criteria. During the index surgery, 209 patients $(1.1 \%)$ had anterior vitrectomy and were also excluded in accordance with the methods of the US study. Thus, 19043 patients remained for analysis. Both extracapsular cataract extractions (ECCE) and intracapsular cataract extractions (ICCE) were studied. However, most attention has been given to the current ECCE technique with intraocular lens (IOL) implant.

All hospital admissions experienced by the cataract patients between 1 January 1980 and 1 January 1992 were identified in the DNPR. In this way, a complete history of admissions was created for all index cases, beginning 5-7 years before their index surgery and continuing 4-6 years beyond. Since the exact date of index surgery is not available in the register, the date of index admission was used instead. The index cases were followed until the detection of an episode of $\mathrm{RD}$, any other intraocular procedure unrelated to an $\mathrm{RD}$ repair, death, or the end of the study period. An episode of $\mathrm{RD}$ was assumed based on an admission with a diagnosis of RD. Dates of death were obtained by checking all index cases in the Central Danish Person Register using the person ID number.

\section{COMPARABIIITY BETWEEN THE US AND THE}

DANISH STUDY

To obtain Danish results that subsequently could be compared with those of the US National Study of Cataract Outcomes, the definitions of events and the design of the data analysis in the Danish study emulated the US study as closely as possible. The US study is described in detail elsewhere ${ }^{7}$ but in summary it was based on 327666 cataract extractions experienced by Medicare beneficiaries in 1984 with a minimum of 3 years of follow up. Data were obtained from the Medical Provider Analysis and Review (MEDPAR) file maintained by the Health Care Financing Administration. During the study period, ICD-9 codes were used in the MEDPAR file. The codes used in the US study were translated by the Danish team into the corresponding Danish ICD-8 codes. Classification problems were clarified through a personal communication with members of the US team.
During the study period, there was no separate Danish code indicating phacoemulsification. These surgeries are reported as ECCE in the Danish sample. Exclusion of patients as a result of combined procedures during index admission is consistent with the US study design. In the USA, an episode of RD was determined based on an admission with either a diagnosis of $\mathrm{RD}$ or a procedure for repair of $R D$. The latter criterion was not possible in the DNPR since the Danish codes for treatment of the retina were not sufficiently specific. In Denmark, an episode of $\mathrm{RD}$ was determined exclusively based on a coded diagnosis of RD.

DANISH REFERENCE GROUP

To compare the risk of $\mathrm{RD}$ in the cataract group with that in patients not undergoing intraocular surgery, a reference group was created. A sample of 7876 individuals born in Denmark between 1896 and 1935 who were still alive on 1 January 1986 was drawn from the National Person Register. The birth cohort was randomly sampled within each calendar year. Based on their person ID numbers, a history of admissions was created as described for the cataract group. Two hundred forty people with an episode of cataract extraction or $\mathrm{RD}$ recorded in the DNPR before 1 January 1986 were excluded, leaving 7636 individuals for analysis. Starting 1 January 1986, these individuals were followed for 4 years, or until either the detection of an episode of $\mathrm{RD}$, death, or an admission for another intraocular procedure.

\section{STATISTICAL METHODS}

Kaplan-Meier analysis was used for calculating the cumulative risk of $R D .^{10}$ Relevant subgroups were compared by the log rank test. ${ }^{10}$ The relative importance of age, sex, and type of surgery as prognostic indicators was evaluated using the proportional hazards regression method (Cox regression). ${ }^{11}$ A $5 \%$ level of significance was used and $95 \%$ confidence intervals (CI) were calculated when appropriate. For comparison of categorical variables the $\chi^{2}$ test was used. The incidence density of $\mathrm{RD}$ per person year in the reference group was calculated based on the 4 year cumulative risk of $\mathrm{RD}$ experienced by the group. ${ }^{12}$ For each year following the index surgery, a standardised incidence ratio was calculated between the number of $\mathrm{RD}$ observed in the cataract group and the number of $R D$ that would have been expected if the incidence density had been equivalent to the reference group. SAS version 6.09 was used for analysis.

The study was approved by the medical ethics committee for Copenhagen, Denmark.

\section{Results}

Demographic and basic clinical information of the total Danish sample, the ECCE with IOL subgroup, and the Danish reference group is shown in Table 1.

The cumulative 4 year risk of $\mathrm{RD}$ was calculated for the four different types of surgery observed in the Danish sample of patients 50 years or older. The ECCE without IOL subgroup had the highest risk $(3.20 \%$, CI 
Table 1 Demographic and clinical characteristics of $(A)$ the total Danish cataract sample, $(B)$ the ECCE+IOL subgroup, and $(C)$ the reference group

\begin{tabular}{|c|c|c|}
\hline & No (\% of total) & No with $R D$ (\% of strata) \\
\hline \multicolumn{3}{|l|}{ (A) Total Danish cataract sample: } \\
\hline Total & $19043(100.0)$ & $231(1.2)$ \\
\hline ICCE - IOL & $2609(13.7)$ & $52(2.0)$ \\
\hline $\mathrm{ICCE}+\mathrm{IOL}$ & $4555(23.9)$ & $66(1.4)$ \\
\hline ECCE - IOL & $1386(7.3)$ & $36(2.6)$ \\
\hline ECCE + IOL (details in (B) below) & $10493(55.1)$ & $77(0.7)$ \\
\hline \multicolumn{3}{|l|}{ (B) ECCE + IOL subsample: } \\
\hline Total & $10493(100.0)$ & $77(0.72)$ \\
\hline \multicolumn{3}{|l|}{ Sex: } \\
\hline Male & $3651(34.8)$ & $41(1.1)$ \\
\hline Female & $6842(65.2)$ & $36(0.5)$ \\
\hline \multicolumn{3}{|l|}{ Age (years): } \\
\hline $50-59$ & $639(6.1)$ & $11(1.7)$ \\
\hline $60-69$ & $1900(18.1)$ & $25(1.3)$ \\
\hline $70-79$ & 4341 (41.4) & $27(0.6)$ \\
\hline $80-89$ & $3285(31.3)$ & $14(0.4)$ \\
\hline$\geqslant 90$ & $328(3.1)$ & $0(0.0)$ \\
\hline \multicolumn{3}{|l|}{ (C) Danish reference group: } \\
\hline Total & $7636(100.0)$ & $16(0.2)$ \\
\hline \multicolumn{3}{|l|}{ Sex: } \\
\hline Male & $3348(43.9)$ & $4(0.23)$ \\
\hline Female & $4272(56.1)$ & $12(0.11)$ \\
\hline \multicolumn{3}{|l|}{ Age (years): } \\
\hline $50-59$ & $1942(25.5)$ & $3(0.15)$ \\
\hline $60-69$ & $2194(28.8)$ & $6(0.27)$ \\
\hline $70-79$ & $1898(24.9)$ & $4(0.21)$ \\
\hline$\geqslant 80$ & $1586(20.8)$ & $3(0.19)$ \\
\hline
\end{tabular}

$\mathrm{ECCE}=$ extracapsular cataract extraction; $\mathrm{ICCE}=$ intracapsular cataract extraction; $\mathrm{RD}=$ retinal detachment.

Table 2 Standardised incidence ratio (SIR) between observed and expected number of retinal detachments $(R D)$ in the ECCE with IOL subgroup compared with the reference group

\begin{tabular}{llllll}
\hline $\begin{array}{l}\text { Year after } \\
\text { index surgery }\end{array}$ & Person years & $\begin{array}{l}\text { Observed } \\
\text { cases of } R D\end{array}$ & $\begin{array}{l}\text { Expected cases } \\
\text { of } R D\end{array}$ & SIR & $(95 \%$ CI) \\
\hline 1st & 9319 & 44 & 2.704 & 16.27 & $(11.82-21.84)^{\star}$ \\
2nd & 7058 & 11 & 2.048 & 5.37 & $(2.68-9.61)^{\star}$ \\
3rd & 5541 & 10 & 1.608 & 6.22 & $(2.98-11.44)^{\star}$ \\
4th & 4690 & 6 & 1.361 & 4.41 & $(1.62-9.60)^{\star}$ \\
5th & 3111 & 2 & 0.903 & 2.22 & $(0.27-8.00)$ \\
6th & 1374 & 3 & 0.399 & 7.52 & $(1.55-21.99)^{\star}$ \\
\hline${ }^{\star} \mathrm{p}<0.05$. & & & & &
\end{tabular}

2.09-4.31) followed by ICCE without IOL (2.37\%, CI 1.70-3.04), ICCE with anterior chamber IOL $(1.81 \%$, CI $1.34-2.28)$, and ECCE with IOL $(0.93 \%$, CI $0.71-1.16)$ (Fig 1). In pairwise comparisons, the risk in the ECCE with IOL subgroup was significantly lower than the risk in any other subgroup $(\mathrm{p}<0.0003)$.

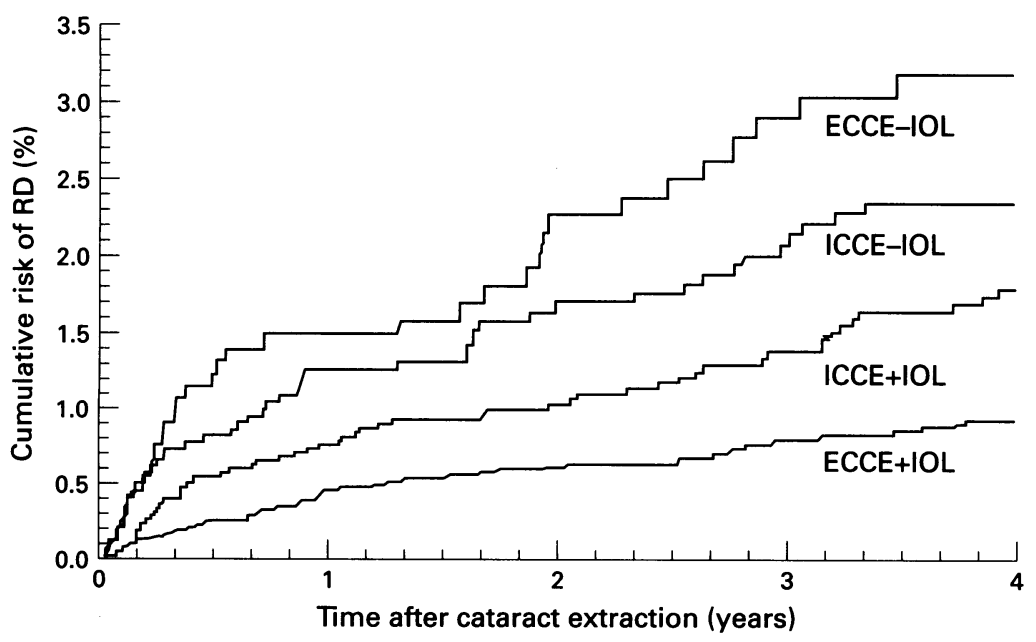

Figure 1 Cumulative risk of retinal detachment (RD) following cataract extraction according to surgical technique in a Danish cataract sample. ICCE=intracapsular cataract extraction, $E C C E=$ extracapsular cataract extraction, $I O L=$ intraocular lens.
Table 3 Estimated 4 year cumulative risk of retinal detachment following extracapsular cataract extraction with IOL implant

\begin{tabular}{lll}
\hline Subgroup & $\begin{array}{l}\text { Cumulative 4 year } \\
\text { risk (\%) }\end{array}$ & $(95 \%$ CI) \\
\hline Sex ${ }^{\star}:$ & & \\
Male & 1.60 & $(1.09-2.11)$ \\
Female & 0.59 & $(0.38-0.81)$ \\
Age (years): & & \\
$50-59$ & 2.43 & $(0.97-3.87)$ \\
$60-69$ & 1.51 & $(0.87-2.15)$ \\
$70-79$ & 0.82 & $(0.48-1.15)$ \\
$\geqslant 80$ & 0.47 & $(0.20-0.73)$ \\
\hline
\end{tabular}

* Log rank test, $\mathrm{p}<0.0001$.

The cumulative 4 year risk of $\mathrm{RD}$ was $0.12 \%$ in the reference group of persons with no previous intraocular surgery and the corresponding incidence density was $0.029 \%$ per person year. For all the cataract surgery groups, the annual additional risk of $\mathrm{RD}$ was highest during the first year. For the ECCE with IOL subgroup, 77 cases of $R D$ were seen within the observation period. Of these, 22 cases $(29 \%)$ occurred 2-6 years after the index surgery. In the sixth year after index surgery, the incidence density of RD was still 7.5 times higher than in the reference group $(p<0.05)$ (Table 2$)$.

When looking at the ECCE with IOL subgroup, univariate analysis showed a significantly higher 4 year cumulative risk for men compared with women (Table 3 ). Also young age was significantly associated with a higher 4 year cumulative risk of RD (Table 3 ).

To evaluate the relative importance of age, sex, and type of surgery, we used Cox's proportional hazards model. The current ECCE technique with IOL was, in this analysis, compared with ICCE regardless of implant (Table 4). As in the univariate analyses, a significantly lower relative risk of $\mathrm{RD}$ was observed for women and for those in higher age cohorts. The relative risk of $R D$ following ECCE with IOL was about half the risk following ICCE plus or minus IOL.

Neither the data from DNPR nor the claims data used in the US study include any indication of which eye was involved. Consequently, if a subsequent intraocular surgery is performed, one cannot identify which eye is involved. To avoid this problem, censoring was done in both studies at the time of a second intraocular procedure. However, a patient might have had a cataract extraction or an other intraocular surgery even before the index surgery. Because information on the Danish patients was available 5-7 years before the index surgery, the magnitude of this potential error could be evaluated. When we excluded patients with cataract or other intraocular surgery from 1980 until the time of the index admission, the 4 year risk of $\mathrm{RD}$ following an ECCE with IOL dropped minimally from the above reported $0.93 \%$ to $0.92 \%$ (CI $0.68-$ 1.16).

COMPARISON OF THE DANISH RESULTS WITH THE US NATIONAL STUDY OF CATARACT OUTCOMES In the US national study, only patients age 65 years or older could be included. In a Danish subgroup of cataract patients aged 65 or older 
Table 4 Relative risk ( $R R$ ) of retinal detachment between different levels of age, sex, and type of surgery calculated in a proportional hazard regression model (Cox) for a Danish and a US sample of cataract patients (US data from favitt)

\begin{tabular}{|c|c|c|c|c|}
\hline \multirow[b]{2}{*}{ Variable } & \multicolumn{2}{|l|}{ Danish sample } & \multicolumn{2}{|c|}{ US sample } \\
\hline & $R R(95 \% C I)$ & $p$ Value & $R R$ & p Value \\
\hline \multicolumn{5}{|l|}{ Age (years): } \\
\hline $50-59$ & 1.00 & ref & - & - \\
\hline 60-69 (US 65-69) & $0.88(0.54-1.45)$ & 0.6 & 1.00 & ref \\
\hline $70-79$ & $0.53(0.33-0.86)$ & 0.01 & 0.63 & $<0.0001$ \\
\hline $80-89$ & $0.29(0.17-0.51)$ & $<0.0001$ & 0.27 & $<0.0001$ \\
\hline$\geqslant 90$ & $0.17(0.04-0.72)$ & 0.02 & 0.07 & $<0.0001$ \\
\hline \multicolumn{5}{|l|}{ Sex: } \\
\hline Male & 1.00 & ref & 1.00 & ref \\
\hline Female & $0.63(0.48-0.84)$ & $<0.001$ & 0.60 & $<0.0001$ \\
\hline \multicolumn{5}{|l|}{ Type of surgery: } \\
\hline ICCE & 1.00 & ref & 1.00 & ref \\
\hline ECCE/phaco & $0.46(0.35-0.61)$ & $<0.001$ & 0.53 & $<0.0001$ \\
\hline
\end{tabular}

slightly more males were observed compared with the US sample (33.2\% versus $31.7 \%$ ), and more intracapsular surgery was performed in Denmark (38.5\% versus $30.8 \%$ ). Of the Danish ECCE, $11.3 \%$ of surgery was performed without an IOL implant. The fraction performed as ECCE without IOL was not reported from the US study. The DNPR has no data on race but approximately $99 \%$ of the studied age group is white compared with $90.4 \%$ in the US sample. The mean age for a Danish subgroup of patients 65 years and over was 77.6 years versus 76.6 years in the US sample. The rate of anterior vitrectomy during simple cataract extraction was $1.3 \%$ in the Danish and $1.1 \%$ in the US sample ( $p>0.05)$

In the US study, no distinction between an extraction with and without IOL was made. Replicating this categorisation on a Danish subsample of patients 65 years or older, we found a 4 year cumulative risk for ICCE of $1.80 \%$ (CI 1.41-2.19) compared with $1.55 \%$ in the US sample. In the US study, the 4 year cumulative risk for the ECCE group was $0.90 \%$. If only cases of ECCE with IOL were included in the Danish sample, a 4 year cumulative risk of $0.77 \%$ (CI $0.55-0.99$ ) was observed and if all cases of ECCE regardless of implant were included, a risk of $0.98 \%$ (CI 0.75-1.21) was observed. In both the Danish and the US studies the difference in risk of $R D$ between the ICCE and the ECCE groups is highly significant $(p<0.0001)$.

For the proportional hazards model, we included all patients 50 years or older to obtain maximum information and statistical power (Table 4). However, if only patients 65 years or older were included in the Danish regression model, we saw only trivial changes in the results (data not shown). All the relative risks between subgroups reported from the US study were similar to and within the confidence intervals for the corresponding relative risks observed in the Danish sample.

\section{Discussion}

We have obtained results applicable to the Danish public health care system regarding the risk of retinal detachment (RD) following cataract surgery. Through a uniformity in the definition of events and the planning of analysis, it was subsequently possible to conduct a reliable comparison of this outcome of cataract surgery in Denmark with surgery performed in the USA. Similar cumulative risks of $\mathrm{RD}$ were shown. The US cumulative 4 year risk was $0.90 \%$ for the conjoint group of ECCE with and without IOL. This is very close to and within the confidence interval of the Danish risk of $0.98 \%$ for the corresponding Danish age group. However, because the ECCE technique had been used for a longer time in the USA, the proportion of ECCE without IOL might have been lower in the USA than the $11.3 \%$ seen in Denmark. In a US claims data study including cataract patients between 1986-7, the proportion of ECCE without IOL implant was $2 \% .{ }^{13}$ Even when looking at the Danish risk of $0.77 \%$ for ECCE with IOL only, the US risk is still similar and within its confidence interval. The rates of anterior vitrectomy at the time of the surgery were also quite similar. No difference in the outcomes of surgery for cataract patients has thus been observed between the two health care systems regarding the proportion of intraoperative anterior vitrectomy and the postoperative risk of $\mathrm{RD}$.

Previous studies of $\mathrm{RD}$ following ECCE have suggested rates of $0.4 \%$ to $3.6 \% .^{1-6}$ The incidence of $0.029 \%$ per person year in the Danish reference group is very similar to that of $0.021 \%$ previously reported from a Finnish population of people 60 years or older. ${ }^{14}$ Several risk factors were significantly associated with the risk of RD. The association with age and sex has previously been reported in other studies. ${ }^{146}$ The relative risks estimated in the Danish and US studies were strikingly similar.

Others have reported that only a few cases of RD were seen beyond 1-2 years after cataract extraction. ${ }^{156}$ However, in these studies a case of $\mathrm{RD}$ would only be detected if the patient returned to the clinic that originally performed the cataract surgery. The nationwide status of the DNPR leaves only minimal losses to follow up possible as discussed later. In the Danish study, $29 \%$ of the identified episodes of RD in the ECCE with IOL group were seen more than 2-6 years after surgery. In the sixth year after index surgery, a 7.5 times higher incidence of $\mathrm{RD}$ was observed compared with the reference group (Table 2). If this elevated risk of $R D$ continues beyond the study observation period, the cumulative risk of $\mathrm{RD}$ experienced by a younger cataract surgery patient would be expected to increase to quite a high level in the following decades of life. This finding might suggest a more restrictive attitude towards early cataract surgery. Similarly, this finding has obvious implications for those considering clear lens extraction as a refractive procedure.

The Danish reference group was created to estimate the risk of RD in a patient who did not have cataract surgery. The cataract surgery group and the reference group might not have been completely comparable as the reference group might have included some patients with retinal comorbidity contraindicating cataract surgery. These patients might have a higher risk of RD. However, this would if anything tend towards an underestimation of the differ- 
ence between the cataract group and the reference group and, consequently, not affect the conclusion of the study.

\section{POTENTIAL SOURCES OF ERRORS}

The accuracy of administrative data depends on the care with which the activity of an admission is observed, classified, and coded. In a review of 1094 admissions, the DNPR found no clinically relevant variation between three observers in $90.1 \%$ of the reviewed diagnoses in general surgery. For the procedure codes no clinically relevant variation was seen for $91.4 \%$ of the admissions. ${ }^{15}$ Of the reported dates, $99.9 \%$ were correct within 3 days. To evaluate the completeness of reporting to the DNPR, we compared the total number of cataract extractions found in the DNPR with the result of a national survey performed by the Danish Ophthalmological Society. ${ }^{16}$ For 1987 , a difference in numbers of $4.2 \%$ was observed between the survey and the DNPR file. For one hospital (Hvidovre University Hospital) we also examined the local surgical record. We observed a difference in numbers of $0.6 \%$ between the local registration and the DNPR file for the year 1986. Based on these findings, we believe the reliability of the diagnosis and procedure codes in the DNPR to be acceptable for the present purpose.

Only information on inpatient procedures in public hospitals is contained in the Danish data base. However, we believe that the results represent the Danish experience, since outpatient procedures and surgery at private clinics comprised only $5 \%$ of all cataract surgery during the study period. ${ }^{9}$ The US study was initially done entirely for inpatient cataract surgery, but a subsequent US study of outpatient surgery showed similar results. ${ }^{17}$

There is a possible ascertainment error since the detection of a case of $R D$ requires that the case was treated in a hospital. However, no cases of RD would have been treated on an outpatient basis in Denmark during that period. Some cases of $\mathrm{RD}$ with a very poor prognosis might have been left untreated and not referred to a hospital leading to an underestimation of the risk. However, we also expect this number to be small in both Denmark and the USA. The nationwide status of the DNPR leaves no further losses to follow up possible, except emigration, which is rare in the studied age groups.

A potential error is introduced because neither the Danish nor the US data can identify which eye underwent surgery. This error was minimised by censoring at the time of a second cataract surgery or another intraocular surgery. If a patient has already had a first eye cataract surgery at the time of index admission there is still potential for confusion. However, as shown in this study no change in the risk of RD was seen when we eliminated this potential error by excluding all patients who had a cataract or another intraocular surgery performed in the 7 years before their index surgery. Based on this, it seems justified to conclude that the error introduced by the missing indication of laterality in the databases is of minimal impact.

A comparison of the US and Danish results relies on an assumption of a comparable definition of events and analysis. Great care was taken in the translation and the comparison of codes for events but some differences could not be avoided. During the study period, phacoemulsification was not coded separately in the DNPR but was categorised as an ECCE. However, very few phacoemulsification procedures were performed in Denmark at that time. Consequently, the Danish ECCE group is still comparable with the US ECCE group. The lack of specificity for surgical codes for $\mathrm{RD}$ treatment in the Danish coding system made it necessary to define a case of $\mathrm{RD}$ based on a diagnosis of $R D$. This is different from the US study where a case of $\mathrm{RD}$ was defined based on either a diagnosis or a procedure for RD. However, we have no reason to believe that any major systematic errors in the coding practice exist that could lead to a significant number of unreported cases of RD in the Danish sample.

Lists of all codes for diagnoses and procedures are available from authors on request.

This study has been supported by the Agency for Health Care and Policy Research, USA (HS07085), the Danish Eye Research Foundation, the John and Birthe Meyer Foundation (Denmark), Denmark Health Foundation, the Health Insurance Fond (Denmark), and the Danish Medical Research Council.

1 Smith PW, Stark WJ, Maumenee AE, Enger CL, Michels RG, Glaser BM, Bonham RD. Retinal detachment after extracapsular cataract extraction with posterior chamber intraocular lens. Ophthalmology 1987;94:495-504.

2 Coonan P, Fung WE, Webster RG, Allen AW, Abbott RJ. The incidence of retinal detachment following extracapsular cataract extraction. Ophthalmology 1985;92:1096-101.

3 Wilkinson CP, Anderson LS, Little JH. Retinal detachment following phacoemulsification. Mod Probl Ophthalmol 1977; 18:503-7.

4 Nielsen NE, Naeser K. Epidemiology of retinal detachment following ECCE: a follow-up study with an analysis of risk factors. $\mathcal{F}$ Cataract Refract Surg 1993;19:675-80.

5 Everett WG, Hurite FG, Sorr EM. Retinal detachment following cataract extraction by phaco emulsification. Mod Probl Ophthalmol 1977;18:503.

6 Hünemohr D, Pham DT, Wollensak J. Netzhautablösung bei hinterkammerlinse. Klin Monatsbl Augenheilkd 1992; 200:91-4.

7 Javitt JC, Vitale S, Canner JK, Krakauer H, McBean AM, Sommer A. National outcomes of cataract extraction I. Retinal detachment after inpatient surgery. Ophthalmology Retinal detachment

8 The international classification of diseases: 9th revision, clinical modifications. ICD-9CM. 3rd ed. Vol 1. Diseases tabular list. Washington DC: US Department of Health Human Services, 1989

9 Sigmund H, Danneskiold-Samso B. Cataract surgery at hospitals and private clinics. Danish Hospital Institute, Report 92.01, 1992, Copenhagen, Denmark (in Danish).

10 Altman D. Practical statistics for medical research. London: Chapman and Hall, 1991.

11 Cox DR. Regression models and life tables. $f R$ Stat Soc 1972;B34:187-220.

12 Kleinbaum DG, Kupper LL, Morgenstern H. Epidemiologic research. Principles and quantitative methods. New York: Van Nostrand Reinhold Company, 1982.

13 Javitt JC, Tielsch JM, Canner JK, Kolb MM, Sommer A, Steinberg EP. Increased risk of retinal complications associated with ND:YAG laser capsulotomy. Ophthalmology 1992;99:1487-98.

14 Laatikainen L, Tolppaanen E-M, Harju H. Epidemiology of rhegmatogenous retinal detachment in a Finnish population. Acta Ophthalmol 1985;63:59-64.

15 Danish Board of Health. Evaluation of The National Patient Register. Sygehusstatistik II:57:1993.Copenhagen, Denmark (in Danish).

16 Danish Ophthalmological Society. Future eye-care in Denmark. Copenhagen, Denmark 1990 (in Danish).

17 Javitt JC, Street DA, Tielsch JM, Wang Q, Kolb MM, Javitt JC, Street DA, Tielsch JM, Wang Q, Kolb MM,
Schein OD, et al. National outcomes of cataract extraction . Retinal detachment and endophthalmitis after outpatient cataract surgery. Ophthalmology 1994;101:100-6. 